\title{
Trends and Predictors of 30-day Readmission Among Patients Hospitalized with Infective Endocarditis in the United States
}

Deepak Kumar Pasupula ${ }^{1}$, Anusha Ganapati Bhat ${ }^{2}$, Sudeep K. Siddappa Malleshappa ${ }^{2}$, Amir Lotfi $^{3}$, Mara Slawsky ${ }^{3}$, Sam Buffer ${ }^{4}$, Quinn Pack ${ }^{3}$, Samir Saba ${ }^{4}$

1. Internal Medicine, University of Pittsburgh Medical Center, Pittsburgh, USA 2. Internal Medicine, Baystate Medical Center, Springfield, USA 3. Cardiology, Baystate Medical Center, Springfield, USA 4. Cardiology, University of Pittsburgh Medical Center, Pittsburgh, USA

$\square$ Corresponding author: Deepak Kumar Pasupula, 84pdeepak@gmail.com Disclosures can be found in Additional Information at the end of the article

\section{Abstract}

\section{Background}

The incidence and 30-day readmission rates of patients with infective endocarditis (IE) are not fully determined. We used the United States Nationwide Readmission Database (NRD) to assess national trends and predictors of 30-day readmission.

\section{Methods}

We queried the NRD from 2010 to 2014 and identified patients with index hospitalizations primarily for IE. Univariate and multivariate logistic regression analyses were conducted to identify predictors of 30-day readmission.

\section{Results}

A total of 48,500 patients (mean age $58 \pm 19$ years; $38 \%$ women; $6.4 \%$ died during index hospitalization) were admitted for IE. There was an annual decrease in hospitalization rates by 1.5\%. With an exception for 2014, subsequent 30-day readmission rates remained relatively unchanged. All-cause 30-day readmission occurred in $25.4 \%$ of patients, $21.8 \%$ of which were due to acute or subacute bacterial endocarditis. Leaving against medical advice (odds ratio (OR): 3.46, 95\% CI: 3.12 - 3.84; P <0.001), history of drug abuse and a cardiac implantable electronic device in situ (OR: 2.17, 95\% CI: 1.53 - 3.08; P <0.001), fungal IE (OR: 1.5, 95\% CI: 1.28 - 1.76; $\mathrm{P}<0.001$ ), and uninsured patients (OR: 1.39, 95\% CI: $1.12-1.74, \mathrm{P}<0.001$ ) were the strongest independent predictors of 30-day readmission. Readmission cost (\$58 million annually) accounted for $14 \%$ of the total hospitalization cost.

\section{Conclusions}

The annual incidence of IE in the US decreased slightly from 2010 to 2014, but the 30-day readmission rates remained relatively unchanged. Addressing modifiable predictors of readmission may reduce the financial burden of IE on health care.

Categories: Cardiology, Internal Medicine, Infectious Disease

Keywords: infective endocarditis, 30 day readmission, predictors of readmission, elixhauser 


\section{Introduction}

Infective endocarditis (IE) is a lethal disease, with nearly $25 \%$ mortality at six months [1] and $18 \%$ within one year of discharge from index hospitalization [2]. The incidence of IE is estimated at approximately 15 cases per 100,000 people in the United States (US) and is believed to be increasing [3]. A study from the Danish registry in 2011 demonstrated high rates of readmission to the hospital (65\%) among IE patients, with the majority (85\%) readmitted within one year following discharge [2]. Whether these alarming numbers are specific to the Danish population or whether national trends across the United States mirror them remains unsettled. Due to the paucity of large-scale studies, we conducted the present analysis to assess the trends in incidence of IE admissions, the 30-day readmission rates after index hospitalizations, and the predictors and costs associated with readmissions in the United States using the Nationwide Readmissions Database (NRD).

\section{Materials And Methods}

\section{Data source}

We conducted a retrospective analysis of the NRD from January 1, 2010, to December 31, 2014. The NRD is the largest all-payer annual national database sponsored by the Agency for Healthcare Research and Quality under the Healthcare Cost and Utilization Project (HCUP) [4], representing $49.1 \%$ of the total hospitalizations from 22 states in the United States. The NRD collects deidentified annual discharge data by assigning a unique number to every patient in a given year. Therefore, patients can be tracked across various hospitals in the United States using that number. The NRD data is publicly available from HCUP. We accessed and analyzed these data in compliance with the Health Insurance Portability and Accountability Act (HIPAA) of 1996. This protocol was exempted from the institutional review board's approval.

The NRD provides deidentified patient demographic and clinical variables (e.g., age, sex, race, and comorbidities), variables to assist with a national estimate (discharge weight, stratum used for weighing), and readmission information (verified patient linkage number, length of stay). For each hospitalization, admission diagnoses and procedures are provided using the International Classification of Diseases, Ninth Revision-Clinical Modification (ICD-9-CM) codes for admissions prior to October 1, 2015.

\section{Study population}

We identified all IE index admissions between January 1, 2010, and December 31, 2014, using ICD-9-CM codes 421.0, 421.1, 421.9, 036.42, 098.84, 112.81, 115.14, or 115.94 as primary admission diagnosis (DX1 only). The validity of these codes has been previously published [5]. Patients were excluded from the analysis if their age was < 18 years, if they had missing data, or if they were hospitalized in the month of December because the 30-day readmission during the same calendar year could not be ascertained. Out-of-state admissions also had to be excluded since they would not represent readmission in the State Inpatient Database (SID), which is the building block of the NRD. We computed the Elixhauser Comorbidity Index (ECI) [6], ECI readmission score, and ECI mortality scores [7] using the software provided by the HCUP [8].

\section{Description of variables}

In the study sample, age was stratified into the following groups: 18 - 54, 55 - 64, 65 - 74, 75 84 , and $>85$ years, and ECI was categorized into four subgroups: $0,1,2$, and $>3$, which has been adopted in previous studies [9]. HCUP identified hospitals as teaching if they had an American Medical Association-approved residency program, if it was a member of the Council 


\section{Cureus}

of Teaching Hospitals, or if it had full-time equivalent interns and a resident-to-bed ratio of > 0.25 . Hospital size was classified into small, medium, and large based upon the number of hospital beds. Length of stay (LOS) was stratified into the following clusters: $<2,3-4,5-6$, and $>7$ days.

\section{Infective endocarditis etiology and risk factors}

The causative organisms of IE were identified using primary or secondary ICD-9-CM codes during the index admission and are described in Table 1. Common microorganisms (Staphylococcus species, Streptococcus species, Gram-negative bacilli, and fungus) were identified as previously described [5]. Risk factors for IE were classified into the following categories: (1) history of drug abuse, (2) presence of a cardiac implantable electronic device (CIED) in situ, (3) presence of a prosthetic valve, or (4) the absence of any of these medical conditions (native valve). The ICD-9-CM codes for these medical conditions have been summarized in Table 2.

Demographic Variables

Age Cluster

18 - 54 years

55 - 64 years

$65-74$ years

75 - 84 years

85 - 90 years

Females

Etiology

Staphylococcus species

Streptococcus species

Gram negative bacillus

Fungus

Primary expected payer

Medicare

Medicaid

Private

\section{Readmitted within 30-} days

Not readmitted within 30 days

pvalue

$<$

0.001

5,384

2,395

2,038

1,639

0,849

4,899

$(40 \%)$

(44\%)

15,006

(41\%)

(19\%) $\quad 7,196$

(20\%)

(17\%) $\quad 6,099$

5,266

2,630

13,714
(38\%) 0.001
3,962

4,300

792

230

(2\%)

454

$<$ 0.001 0.001

$\begin{array}{lll}5,813 & (47 \%) & 16,524 \\ 2,234 & (18 \%) & 5,692 \\ 2,689 & (22 \%) & 9,188\end{array}$




\section{Cureus}

Self-pay

No charge

Other $^{a}$

Non-elective admission

Bed size of hospital

Small

Medium

Large

Control/ownership of hospital

Government, nonfederal

Private, non-profit

Private, invest-own

Hospital urban-rural designation

Large metropolitan areas with at least 1 million

residents

Small metropolitan areas with less than 1 million residents

Micropolitan areas

Not metropolitan or micropolitan (non-urban

residual)

Teaching status of urban hospitals

Metropolitan non-teaching

Metropolitan teaching

Non-metropolitan

Disposition

Alive during initial admission

Post-acute care facility

Short-term hospital

Against medical advice

Died during initial admission
979

$(8 \%)$

2,932

152

$(1 \%)$

373

405

$(3 \%)$

1,385

11,329

$(92 \%)$

32,699

001

0.381

1,093

$(9 \%)$

3,281

$(9 \%)$

2,668

$(22 \%)$

7,643

8,543

$(69 \%)$

25,272

$(70 \%)$

0.001

1,658

$(13 \%)$

4,975

(14\%)

8,998

$(73 \%)$

26,971

(75\%)

1,649

$(13 \%)$

4,250

(12\%)

$<$

0.001

7,261

$(59 \%)$

20,367

(56\%)

4,193

$(34 \%)$

13,414

(37\%)

702

(6\%)

1,964

148

(1\%)

452

0.066

3,933

(32\%)

11,975

(33\%)

7,521

(61\%)

21,805

$(60 \%)$

850

(7\%)

2,416

(7\%)

$<$

0.001

$\begin{array}{llll}11,300 & (92 \%) & 33,146 & (92 \%) \\ 3,984 & (32 \%) & 9,413 & (26 \%) \\ 586 & (5 \%) & 1,618 & (4 \%) \\ 847 & (7 \%) & 817 & (2 \%) \\ 0 & (0 \%) & 3,050 & (8 \%)\end{array}$




\title{
Cureus
}

Died during readmission

Cost of hospitalization during index admission (\$)

Cost of hospitalization during readmission (\$)

Length of stay during index admission (days) ${ }^{\mathrm{b}}$

Length of stay during readmission (days) ${ }^{b}$
1,004

36,265

23,535

10

6
10

$(0 \%)$

\section{TABLE 1: Patient demographics stratified by 30 -day readmission}

a Other payer includes worker's compensation, CHAMPUS, CHAMPVA, Title $\mathrm{V}$, and other government programs. b Value expressed as median. $\mathrm{c} p$-value $=0.179$.

CHAMPUS: Civilian Health and Medical Program of the Uniformed Services; CHAMPVA: Civilian Health and Medical Program of the Department of Veterans Affairs

\author{
Microorganism \\ ICD-9-CM codes during the index admission \\ Staphylococcus species \\ 0381, 03810, 03811, 03812, 03819, 0411, 04111, 04112, 04110 and 04119 \\ Streptococcus species \\ 0380, 0382, 0410, 04100, 04101, 04102, 04103, 04104, 04105, 04109 and 0412 \\ Gram negative bacillus \\ 0384, 03840, 03841, 03842, 03843, 03844, 03849, 0413, 0414, 0415, 0416, 0417 and 04185 \\ Fungus \\ $1125,11281,1160,11504,11514,11594$ and 1173
}

\section{TABLE 2: Etiological agent among infective endocarditis patients}

ICD-9-CM: International Classification of Diseases, Ninth Revision-Clinical Modification

\section{Endpoints}

The primary endpoint of the study was to assess the annual trends in IE hospitalizations, 30day readmission rates, and predictors of readmissions. Time to readmission was computed as the difference between index hospitalization discharge and subsequent hospitalization for any cause. If there were multiple readmissions during the 30-day period after the index hospitalization, then only the first readmission was considered. Secondary endpoints included identifying an etiological agent for IE, the top 10 reasons for readmission, and the cost of index and readmission hospitalization. Reasons for readmission were identified using primary diagnosis ICD-9 CM codes for readmission hospitalizations. All ICD-9 CM codes for similar diagnoses were pooled together. The costs associated with the index and readmission hospitalizations were computed in terms of 2014 dollars by using the consumer price index data for medical care released by the United States Department of Labor, Bureau of Labor Statistics [10]. 


\section{Cureus}

\section{Statistical analysis}

Categorical variables are presented as weight values and percentages and compared between patients with versus without readmissions using the $\chi 2$ test. Continuous variables are presented as mean \pm standard error and compared between patients with versus without readmissions using the Wilcoxon rank test. Multivariable logistic regression for age cluster, sex, ECI, mode of acquisition, etiology, LOS, disposition on index admission, and primary payer were conducted to assess independent predictors for 30-day readmission. A linear regression analysis was performed for continuous variables, as appropriate, to evaluate for the significance of trends. Statistical analyses were performed using IBM SPSS version 26.0 (IBM Corp, Armonk, NY, US).

\section{Results}

The study cohort consisted of 21,076 unique patients admitted with a primary diagnosis of IE between January 1, 2010, and November 30, 2014, representing 48,500 observations, after applying discharge weights. Over the study period, there was a slow but steady decline in the number of IE hospitalizations (10,081 (2010) vs 9,331 (2014); $\mathrm{p}<0.001)$ with the incidence decreasing by $1.5 \%$ annually. Trends in IE hospitalization and associated 30-day readmissions are shown in Figure 1.

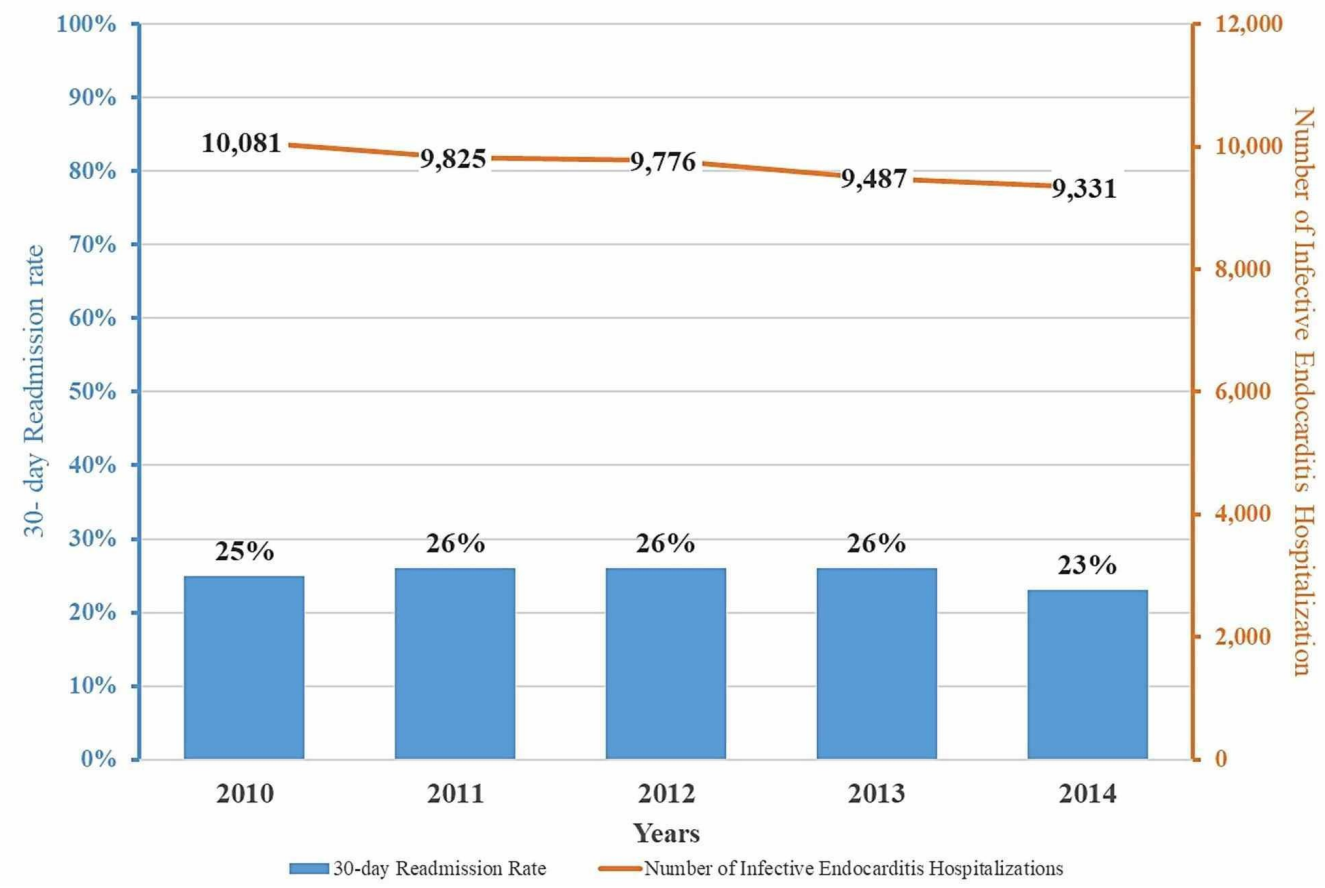

FIGURE 1: Trends in infective endocarditis hospitalization and all-cause 30-day readmission rates

The absolute number of patients hospitalized for infective endocarditis decrease consistently over the study period but all-cause 30-day readmission rates increase slightly until 2013 and then decline in 2014

Table 1 details the demographic and clinical characteristics of IE patients as well as the characteristics of their hospital admission. In the study cohort, the largest proportion of patients admitted with IE belonged to the 18-54 years age group (41\%). The mean age of the overall cohort was $58+19$ years, $38 \%$ were women, and the majority of patients had significant 
comorbidities ( $52 \%$ with ECI $\geqslant 3$ ). Approximately, $46 \%$ of the patients had Medicare as their primary insurance provider. Nearly $58 \%$ of patients were admitted into a metropolitan (area with $\geqslant 1$ million residents) teaching hospital and nearly two-thirds of the hospitalizations were at large, private (non-profit) organizations. In-hospital mortality among all patients admitted with IE was $6.4 \%$.

\section{0-day readmissions}

Among those who survived the initial hospitalization, the 30-day readmission rate for the total cohort was $25.4 \%$. The annual readmission rate was mostly unchanged from 2010 to 2013 but decreased in 2014 by $11.5 \%$ (Figure 1). The time to 30-day readmission after the index IE hospitalization is displayed in Figure 2.

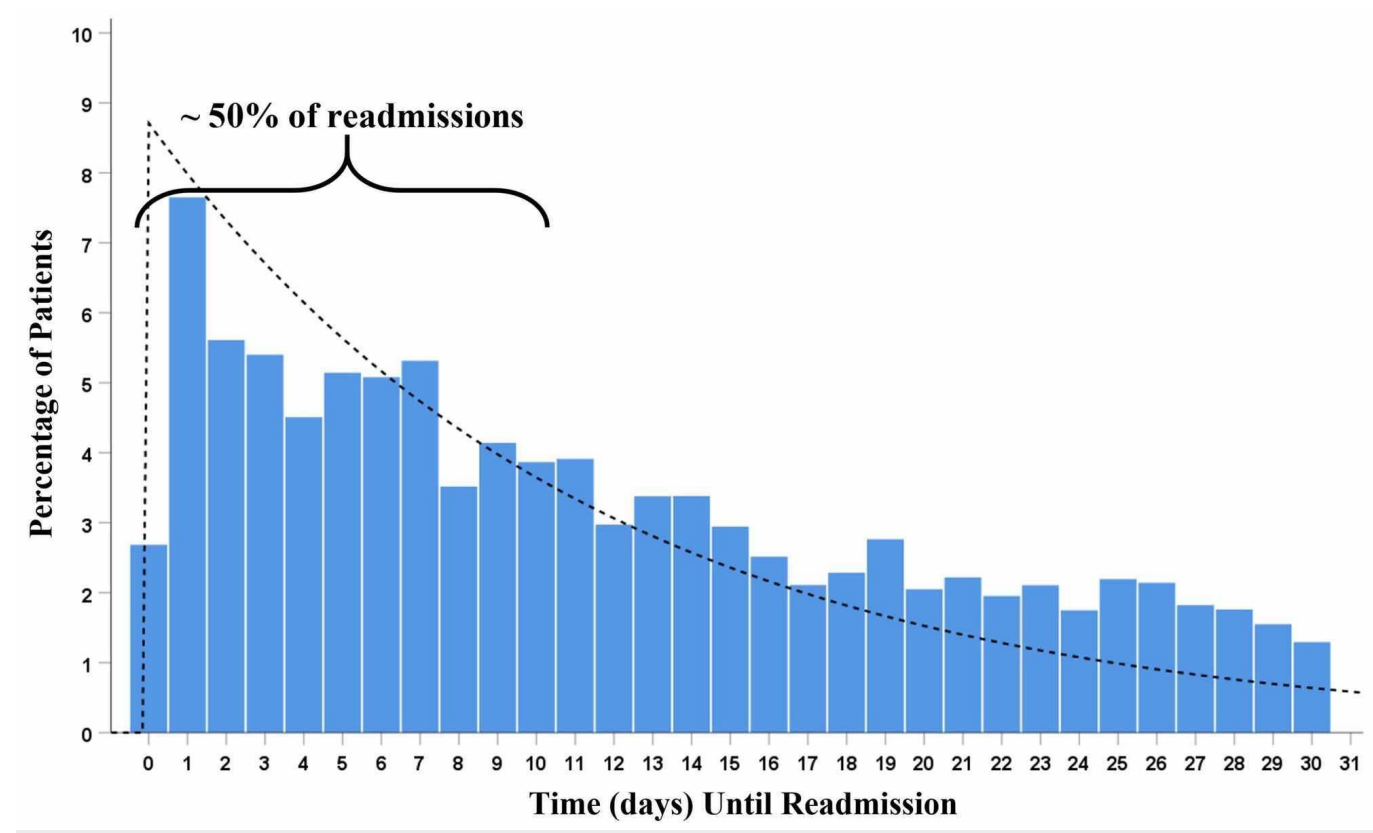

FIGURE 2: Temporal trend in all-cause 30-day readmission of the total cohort after hospitalization for infective endocarditis (exponential curve histogram)

Nearly half of the patient population after being discharged from hospitalization due to infective endocarditis get readmitted in the first 10 days.

The highest readmission rate of $7.6 \%$ was noted within the first 24 hours of discharge. During the index hospitalization, $22.3 \%$ of patients had valve surgery as compared to $12.1 \%$ during readmission. A small number of patients $(n=64,0.1 \%)$ had valve surgeries during both hospitalizations.

The demographics of patients stratified by 30-day readmission have been summarized in Table 2. When compared to those who did not get readmitted, patients readmitted were more likely to be women (40\% vs 38\%, p <0.001), between the age group of 18 and 54 years (44\% vs $41 \%$, p $<0.001$ ), and were admitted emergently (elective admission, $8 \%$ vs $10 \%$; $p<0.001$ ). Hospitals in larger metropolitan areas (more than one million residents) had higher readmission rates (59\% vs $56 \%, \mathrm{p}<0.001$ ) while private non-profit hospitals had less readmissions (73\% vs 75\%, $\mathrm{p}<0.001)$. The readmitted patient had significantly higher ECI $(\geqslant 3)$, as summarized in Table 3. The average ECI readmission score was significantly higher among patients readmitted within 


\section{Cureus}

30 days ( 15 vs $14, \mathrm{p}<0.001$ ). Although the average ECI mortality score was the same in both the groups, there was a $28 \%$ increase in mortality rate among patients who got readmitted within 30 days (6.4\% mortality during index admission vs $8.2 \%$ mortality during readmission, $\mathrm{p}$ $<0.001)$. Among patients who were readmitted, Staphylococcus species, Gram-negative bacilli, and fungal organisms were the most common organisms for IE while Streptococcus species was more common among those who were not readmitted (Table 2).

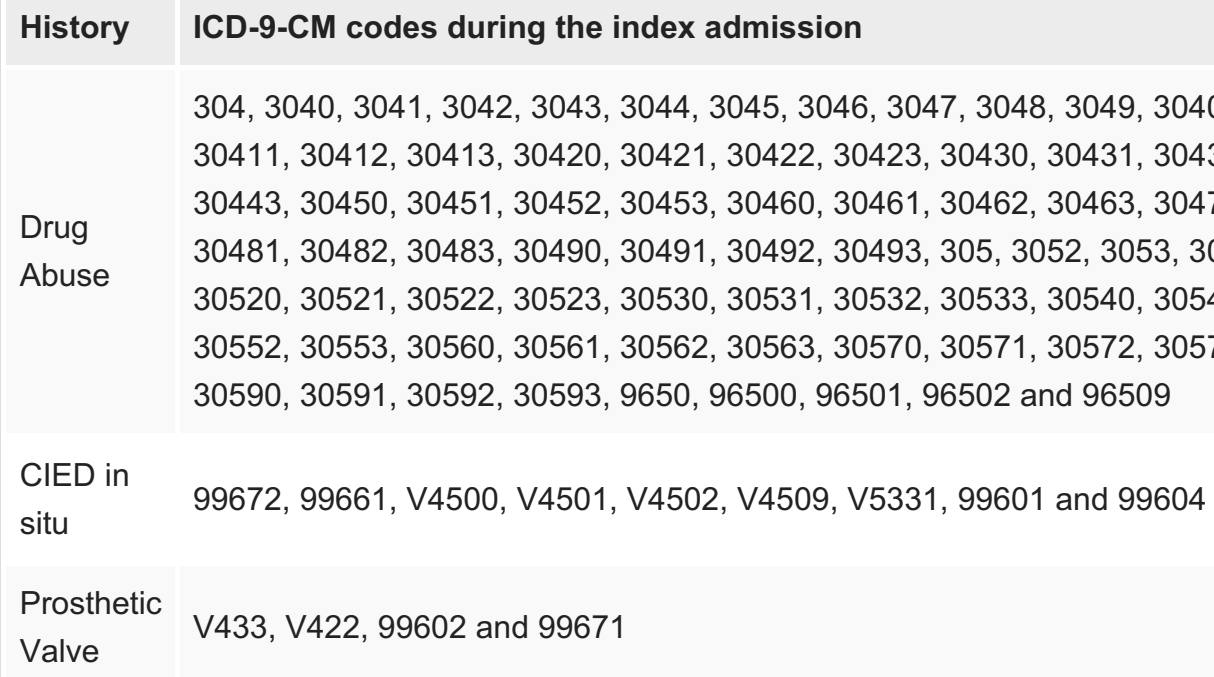

\section{TABLE 3: Risk factors of infective endocarditis}

ICD-9-CM: International Classification of Diseases, Ninth Revision-Clinical Modification; CIED: Cardiac implantable electronic device (pacemaker and defibrillator)

The identity of IE organisms remained relatively stable over the study period, except for the presence of Gram-negative bacilli, which decreased by $9.3 \%$ annually (Table 4). The association of IE with a history of drug abuse increased by $10.6 \%$ annually over the study period while those with native valve endocarditis decreased only by $4.2 \%$ annually. These trends have been summarized in Table 4.

\begin{tabular}{|c|c|c|c|c|c|}
\hline & \multicolumn{2}{|c|}{$\begin{array}{l}\text { Readmitted within } 30 \text { - } \\
\text { days }\end{array}$} & \multicolumn{2}{|c|}{$\begin{array}{l}\text { Not readmitted within } 30- \\
\text { days }\end{array}$} & p-value \\
\hline \multicolumn{6}{|l|}{ Comorbidities } \\
\hline Congestive heart failure & 146 & $(1 \%)$ & 474 & $(1 \%)$ & 0.294 \\
\hline Valvular disease & 111 & $(1 \%)$ & 353 & $(1 \%)$ & 0.472 \\
\hline Pulmonary circulation disease & 166 & $(1 \%)$ & 455 & $(1 \%)$ & 0.432 \\
\hline Peripheral vascular disease & 1,744 & $(14 \%)$ & 4,602 & $(13 \%)$ & $<0.001$ \\
\hline Paralysis & 280 & $(2 \%)$ & 828 & $(2 \%)$ & 0.938 \\
\hline Other neurological disorders & 1,082 & $(9 \%)$ & 3,042 & $(8 \%)$ & 0.181 \\
\hline
\end{tabular}


Cureus

\begin{tabular}{|c|c|c|c|c|c|}
\hline Chronic pulmonary disease & 1,035 & $(8 \%)$ & 2,319 & $(6 \%)$ & $<0.001$ \\
\hline Diabetes without chronic complications & 2,317 & $(19 \%)$ & 6,439 & $(18 \%)$ & $<0.001$ \\
\hline Diabetes with chronic complications & 1,073 & $(9 \%)$ & 2,453 & $(7 \%)$ & $<0.001$ \\
\hline Hypothyroidism & 1,164 & $(9 \%)$ & 3,271 & $(9 \%)$ & 0.159 \\
\hline Liver disease & 1,237 & $(10 \%)$ & 3,073 & $(8 \%)$ & $<0.001$ \\
\hline Peptic ulcer disease with bleeding & 10 & $(0 \%)$ & 30 & $(0 \%)$ & 0.957 \\
\hline Lymphoma & 149 & $(1 \%)$ & 413 & $(1 \%)$ & 0.531 \\
\hline Metastatic cancer & 158 & $(1 \%)$ & 487 & $(1 \%)$ & 0.608 \\
\hline Solid tumor without metastasis & 212 & $(2 \%)$ & 438 & $(1 \%)$ & $<0.001$ \\
\hline $\begin{array}{l}\text { Rheumatoid arthritis/collagen vascular } \\
\text { disorder }\end{array}$ & 408 & $(3 \%)$ & 1,247 & $(3 \%)$ & 0.495 \\
\hline Coagulopathy & 1,876 & $(15 \%)$ & 5,718 & $(16 \%)$ & 0.147 \\
\hline Obesity & 1,290 & $(10 \%)$ & 3,414 & $(9 \%)$ & $<0.001$ \\
\hline Weight loss & 1,417 & $(12 \%)$ & 4,202 & $(12 \%)$ & 0.782 \\
\hline Fluid and electrolyte disorders & 4,849 & $(39 \%)$ & 13,742 & $(38 \%)$ & 0.004 \\
\hline Chronic blood loss anemia & 194 & $(2 \%)$ & 453 & $(1 \%)$ & 0.007 \\
\hline Deficiency anemias & 5,432 & $(44 \%)$ & 14,363 & $(40 \%)$ & $<0.001$ \\
\hline Alcohol abuse & 667 & $(5 \%)$ & 2,028 & $(6 \%)$ & 0.447 \\
\hline Drug abuse & 2,337 & $(19 \%)$ & 6,470 & $(18 \%)$ & 0.005 \\
\hline Psychoses & 655 & $(5 \%)$ & 1,608 & $(4 \%)$ & $<0.001$ \\
\hline Hypertension & 3,461 & $(28 \%)$ & 11,150 & $(31 \%)$ & $<0.001$ \\
\hline Depression & 1,576 & $(13 \%)$ & 3,715 & $(10 \%)$ & $<0.001$ \\
\hline Cardiac & 11,687 & $(95 \%)$ & 34,455 & $(95 \%)$ & 0.344 \\
\hline Human immunodeficiency virus & 75 & $(1 \%)$ & 164 & $(0 \%)$ & 0.032 \\
\hline Elixhauser Comorbidity Index & & & & & $<0.001$ \\
\hline 0 & 719 & $(6 \%)$ & 2,973 & $(8 \%)$ & \\
\hline 1 & 1,850 & $(15 \%)$ & 6,231 & $(17 \%)$ & \\
\hline 2 & 2,976 & $(24 \%)$ & 8,757 & $(24 \%)$ & \\
\hline$>3$ & 6,759 & $(55 \%)$ & 18,235 & $(50 \%)$ & \\
\hline ECI Readmission Score ${ }^{a}$ & $15+0.1$ & & $14+0.06$ & & $<0.001$ \\
\hline $\mathrm{ECI}$ Mortality Score ${ }^{a}$ & $6+0.09$ & & $6+0.05$ & & 0.693 \\
\hline
\end{tabular}




\section{Cureus}

\section{TABLE 4: Elixhauser comorbidity index of patients stratified by $\mathbf{3 0}$-day readmission}

ECl: Elixhauser comorbidity index. a Value expressed as mean (standard error)

The top 10 most common reasons for readmission stratified by year are summarized in Table 5 . The most common cause of readmission was acute on subacute bacterial endocarditis (421.0) and the second most common cause was septicemia (038.9). These remained unchanged during the study period. Infection due to a cardiac device, implant, and graft was the next most common reason for readmission (996.61). Heart failure (acute on chronic systolic heart failure (428.23) and acute on chronic diastolic heart failure (428.33)) steadily increased over the study period as causes for readmission after IE hospitalization. 


\begin{tabular}{|c|c|c|c|c|c|c|c|c|c|c|}
\hline Etiology & \multicolumn{2}{|l|}{2010} & \multicolumn{2}{|l|}{2011} & \multicolumn{2}{|l|}{2012} & \multicolumn{2}{|l|}{2013} & \multicolumn{2}{|l|}{2014} \\
\hline Staphylococcus species & $3,15 r$ & $(31 \%)$ & $3,1 / 2$ & $(32 \%)$ & 3,068 & $(31 \%)$ & 2,813 & $(30 \%)$ & 2,963 & $(32 \%)$ \\
\hline Streptococcus species & 3,867 & $(38 \%)$ & 3,697 & $(38 \%)$ & 3,529 & $(36 \%)$ & 3,491 & $(37 \%)$ & 3,468 & $(37 \%)$ \\
\hline Gram negative bacillus & 669 & $(7 \%)$ & 631 & $(6 \%)$ & 497 & $(5 \%)$ & 520 & $(5 \%)$ & 410 & $(4 \%)$ \\
\hline Fungus & 140 & $(1 \%)$ & 151 & $(2 \%)$ & 150 & $(2 \%)$ & 105 & $(1 \%)$ & 138 & $(1 \%)$ \\
\hline \multicolumn{11}{|l|}{ Risk factors } \\
\hline History of drug abuse & 1,222 & $(12 \%)$ & 1,359 & $(14 \%)$ & 1,656 & $(17 \%)$ & 1,789 & $(19 \%)$ & 2,018 & $(22 \%)$ \\
\hline CIED in situ & 547 & $(5 \%)$ & 525 & $(5 \%)$ & 567 & $(6 \%)$ & 499 & $(5 \%)$ & 423 & $(5 \%)$ \\
\hline History of prosthetic valve & 621 & $(6 \%)$ & 597 & $(6 \%)$ & 666 & (7\%) & 627 & $(7 \%)$ & 588 & $(6 \%)$ \\
\hline History of drug abuse \& CIED in situ & 29 & $(0 \%)$ & 29 & $(0 \%)$ & 28 & $(0 \%)$ & 25 & $(0 \%)$ & 19 & $(0 \%)$ \\
\hline $\begin{array}{l}\text { History of drug abuse \& history of } \\
\text { prosthetic valve }\end{array}$ & 45 & $(0 \%)$ & 82 & $(1 \%)$ & 81 & $(1 \%)$ & 133 & $(1 \%)$ & 103 & $(1 \%)$ \\
\hline $\begin{array}{l}\text { ClED in situ \& history of prosthetic } \\
\text { valve }\end{array}$ & 230 & $(2 \%)$ & 220 & $(2 \%)$ & 188 & $(2 \%)$ & 220 & $(2 \%)$ & 180 & $(2 \%)$ \\
\hline $\begin{array}{l}\text { History of drug abuse \& CIED in situ } \\
\& \text { history of prosthetic valve }\end{array}$ & 15 & $(0 \%)$ & 11 & $(0 \%)$ & 36 & $(0 \%)$ & 39 & $(0 \%)$ & 40 & $(0 \%)$ \\
\hline Native valve & 7,372 & $(73 \%)$ & 7,003 & $(71 \%)$ & 6,555 & $(67 \%)$ & 6,155 & $(65 \%)$ & 5,958 & $(64 \%)$ \\
\hline \multicolumn{11}{|l|}{ Mortality } \\
\hline During index hospitalization & 734 & $(7 \%)$ & 674 & $(7 \%)$ & 629 & $(6 \%)$ & 513 & $(5 \%)$ & 544 & $(6 \%)$ \\
\hline During readmission & 234 & $(9 \%)$ & 234 & $(9 \%)$ & 198 & $(8 \%)$ & 178 & $(7 \%)$ & 159 & $(7 \%)$ \\
\hline
\end{tabular}

\section{TABLE 5: Trends in etiology, risk factors, and mortality among infective endocarditis patients}

CIED: Cardiac implantable electronic device (pacemaker and defibrillator)

In a multivariate regression analysis (Figure 3), the strongest independent predictor for 30-day readmission was leaving the hospital against medical advice (AMA) (relative risk ratio (RR): 3.46, 95\% CI: 3.12 - 3.84; $\mathrm{p}<0.001$ ), followed by patients with a history of drug abuse associated with CIED in situ (RR: 2.17, 95\% CI: 1.53 - 3.08; p <0.001), and patients with a history of drug abuse and prosthetic valve (RR: 1.64, 95\% CI: 1.35 - 1.99; $\mathrm{p}<0.001$ ). A higher ECI was also a strong predictor for readmission (RR: 1.53 , 95\% CI 1.41 - 1.67; p <0.001 for ECI $\geqslant 3$ and RR: 1.41, $95 \%$ CI $1.28-1.54 ; \mathrm{p}<0.001$ for ECI = 2, both compared to ECI = 0). Compared to patients in whom no organisms could be recovered, patients with fungal IE were most likely to be readmitted (RR: 1.5, 95\% CI: 1.28 - 1.76; $\mathrm{p}<0.001$ ), followed by those with Gram negative bacillus IE (RR: 1.22, 95\% CI: 1.12 - 1.33; p < 0.001). Conversely, patients with Streptococcal IE 


\section{Cureus}

(RR: $0.88,95 \%$ CI: $0.84-0.92 ; \mathrm{p}<0.001$ ) had lower 30-day readmission rates. Being uninsured (RR: 1.39, 95\% CI: 1.12 - 1.74, p <0.001), on Medicare (RR: 1.2, 95\% CI: 1.07 - 1.35; p = 0.002), or on Medicaid (RR: 1.34, 95\% CI: 1.19 - 1.52; p < 0.001) were also independent predictors for 30day readmission as compared to other payers.

Risk Factors (vs. Native Valve)

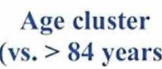

55 - 64 years

65 - 74 years

75 - 84 years

Males (vs. Female)

Elixhauser Comorbidity Index 1 (vs. 0)

History of Drug Abuse

CIED in situ

History of Prosthetic Valve

History of Drug Abuse \& CIED in situ

History of Drug Abuse \& History of Prosthetic Valve

CIED in situ \& History of Prosthetic Valve

History of Drug Abuse \& CIED in situ \& History of Prosthetic Valve

Etiology

Staphylococcus species (vs. no organism) Streptococcus species

Gram negative bacillus

Fungus

Non-elective admission (vs. elective)

Valve surgery during index admission (vs. no surgery)

Length of stay on initial admission $<2$ days (vs. $\geq 7$ days)

3 - 4 days

5 - 6 days

Disposition on initial admission Short term hospital (vs. Home)

Facility

Home health care Against medical advice

Payer Medicare

(vs. Other) Medicaid

Private

Self-pay

No charge

Hospital status

(vs. Non-metropolitan)

Metropolitan teaching

Metropolitan non-teaching

Hospital size Medium

(vs. Small)

Large

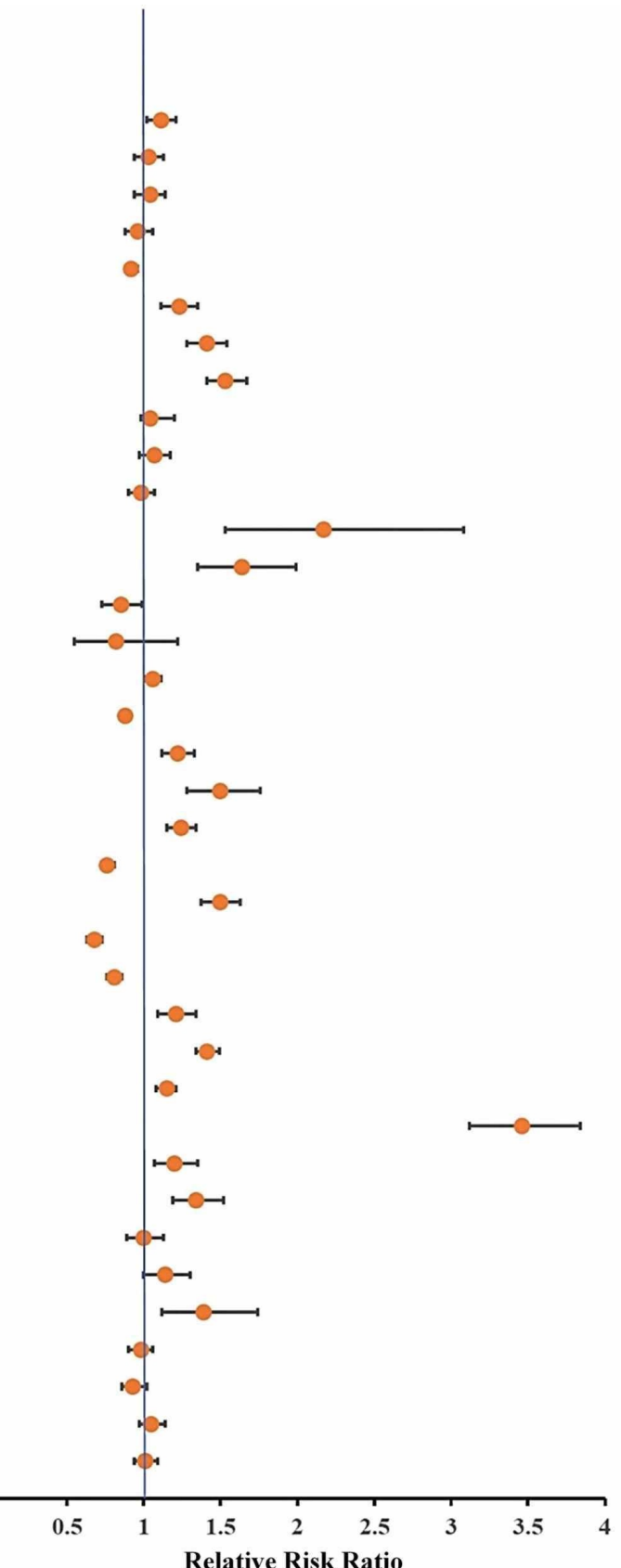

FIGURE 3: Predictors of 30-day readmission among infective endocarditis patients in the United States

The strongest independent predictor for 30-day readmission after hospitalization for infective endocarditis were patients leaving against medical advice, followed by those with a history of drug abuse with a CIED and a history of drug abuse with a prosthetic valve.

CIED: cardiac implantable electronic device 


\section{Cureus}

\section{Cost of hospitalization}

During the study period, the average cost per index hospitalization was $\$ 38,006$, and per readmission, hospitalization cost was $\$ 23,535$. The annual cost for index hospitalization was approximately $\$ 369$ million while for readmission hospitalization, it was $\$ 58$ million. Trends in annual index hospitalization costs and readmission hospitalization costs are summarized in Figure 4. Readmissions accounted for $14 \%$ of the total annual cost incurred toward inpatient care. Medicare and Medicaid together incurred $\$ 38$ million annually towards readmission reimbursement alone, followed by private carriers ( $\$ 13$ million per annum), self-pay ( $\$ 4$ million per annum), other insurance agencies (worker’s compensation, Civilian Health and Medical Program of the Uniformed Services (CHAMPUS), Civilian Health and Medical Program of the Department of Veterans Affairs (CHAMPVA), Title V, and other government programs) ( $\$ 2$ million per annum) and the uninsured (\$0.6 million per annum).

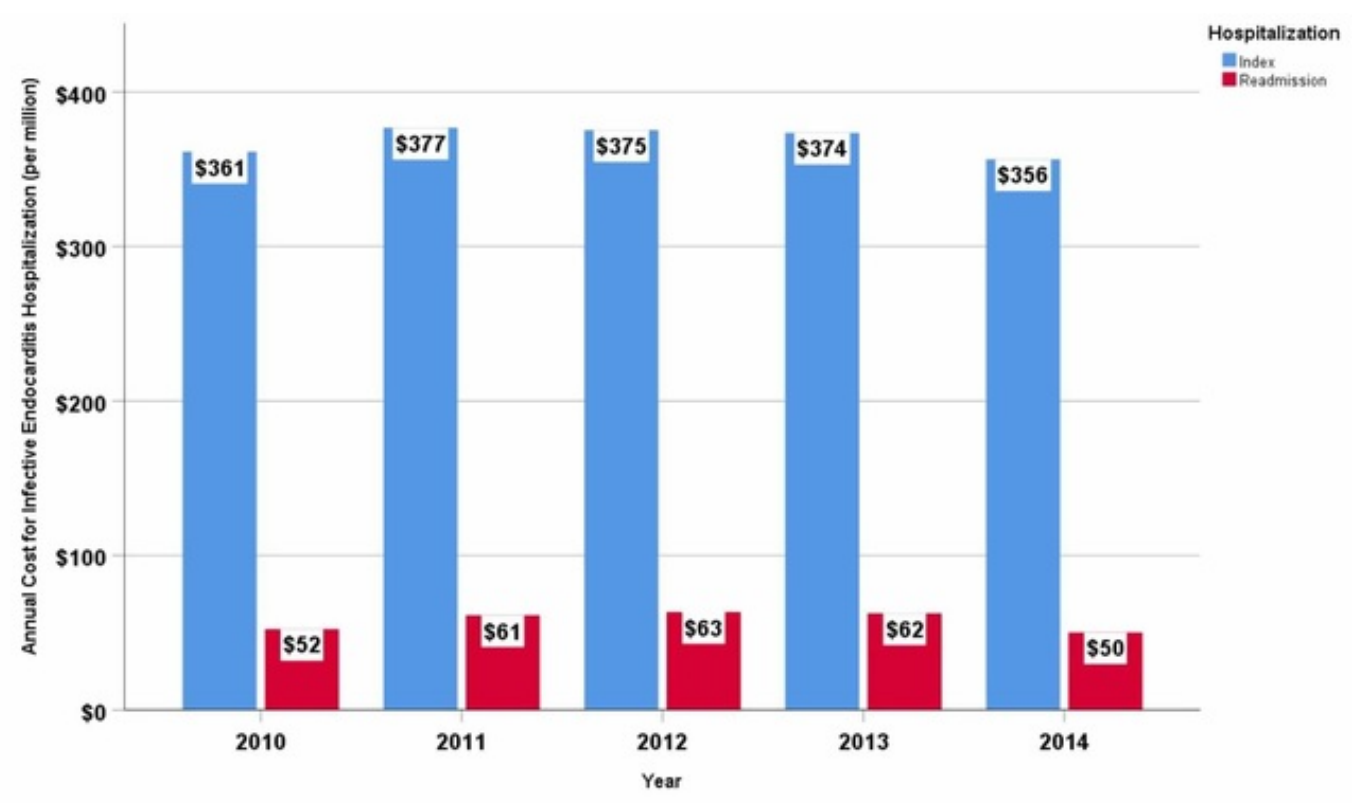

FIGURE 4: Trends-in-annual-cost-(per-million)-for-index-and-30day-readmission-hospitalization-for-infective-endocarditis-inthe-United-States

Infective endocarditis-related index hospitalization and readmission hospitalization cost gradually increased until 2012 and then declined until 2014. Cost of hospitalization is adjusted for inflation by computing in terms of 2014 dollar for medical care.

\section{Discussion}

In this nationwide analysis of IE from the largest available readmission database in the United States, we report a slight decrease in the admission rates for IE and mostly stable 30-day readmission rates annually (except during 2014) from January 1, 2010, to November 30, 2014. The highest readmission rate was within 24 hours of the index hospitalization and nearly half of the patients were readmitted within 10 days after discharge. Acute and subacute bacterial endocarditis was the most common primary diagnoses upon readmission, followed by septicemia and heart failure. Patients leaving the hospital against medical advice and those with a history of drug use in association with implanted cardiac devices or valve prostheses were at the highest risk for readmission in multivariable analyses. Approximately, $\$ 58$ million are spent annually in the US toward IE rehospitalization. 
Appropriate antibiotic therapy and removal of infected prostheses remain the cornerstone therapy in the management of IE. Practice guidelines are well-developed and regularly updated to assist clinicians in choosing the appropriate treatment for their patients [11-13]. Contrary to findings by Pant et al. [14], who documented an earlier increase in hospitalizations for IE, our study demonstrates a slight decline in the incidence of IE after 2010. This may be a real change in trends or possibly explained by essential differences in methodology whereby we used the NRD as opposed to the nationwide inpatient sample. Also, Pant et al. have used total IE admissions in a given year to estimate the incidence of the disease, which could have overestimated the true incidence by counting the same patient multiple times if they had more than one IE admission in the same year. The NRD has been well-validated in previous studies for its accurate representation of national trends in incidences of disease hospitalizations and for its ability to assess readmission rates [15-16].

IE patients have higher all-cause 30-day readmission rate (25.4\%) when compared to the allcause readmission rate for total inpatient stays in 2014 (13.9\% in HCUP statistical brief \# 230) [17]. When compared to the top 20 principal diagnoses with the highest seven-day and 30-day readmission rates in 2014, our data in 2014 showed that IE patients have the highest all-cause seven-day ( $9.4 \%$ vs $9.0 \%$ (the \#1 cause was schizophrenia and other psychotic disorders)) and 30 -day readmission rate ( $23.3 \%$ vs $23.2 \%$ (the \#1 cause was congestive heart failure)). Higher readmission rates among IE patients, when compared to other medical conditions, could not only be associated with higher comorbidities (approximately two-thirds of patients have ECI > 2) but also related to social issues that may lead to suboptimal management and recurring infection. A significant proportion of our IE cohort consisted of patients with a history of drug abuse. This problem, which is reaching epidemic proportions with a $13 \%$ increase in the illicit drug abuse from 2002 to 2013 in the US [18], may impede management plans with patients not only exhibiting non-compliance with treatment but also are likely to be associated with continued high-risk behavior leading to recurrent IE. In light of the recent study by Iversen et al., IE can be treated with oral antibiotics in an outpatient setting among few selected group of patients, which may reduce LOS for index and readmission hospitalizations and possibly allow appropriate or near appropriate management of patients leaving AMA [19]. Despite having the highest readmission rate, IE is not among the six conditions/procedures used by Center for Medicare and Medicaid Services as part of the Hospital Readmission Reduction Program (HRRP) to assess the hospital excess readmission ratio, which is used to penalize hospitals with high readmissions rates [20].

Septicemia (038.9) has remained the second most common primary reason for readmission, which most likely stems from uncontrolled and failed initial therapy from IE bacteremia. It is interesting that previous analyses from our group have shown that septicemia is also the second most common cause for readmission after CIED implantation. It is difficult to tease out, based on our study, if bacteremia leads to infection of the CIED or if IE was due to a poor sterile technique, leading to contamination of the pocket site, which caused septicemia. However, in light of our previous study [15] with a very low incidence of cellulitis or abscess of the chest wall $(0.04 \%)$, we can safely speculate that bacterial seeding caused infection of CIED in situ.

In the multivariate analysis, we demonstrate that patients at higher risk for readmission within 30 days were those who left the hospital AMA, had a history of drug abuse and CIED in situ, had a history of drug abuse and prosthetic valve, having fungal IE, higher comorbidity (ECI >3), and those with a shorter LOS ( $<2$ days). Interestingly, our data demonstrate that it is the combination of risk factors (drug abuse and the presence of CIED or prosthetic valve) that is associated with higher rates of readmission, not each risk factor independently. This double hit finding makes physiological sense since the high-risk behavior leads to repetitive inoculation with infectious agents while the presence of a foreign device or prosthesis allows for the seeding of the infectious agent, leading to recurrence of IE even after adequate therapy. 
This study's major advantage is that it is based on mandatory data reporting from all regions of the 22 states participating in the NRD. These collectively represent the real world of IE across the United States. With patient weights provided by the HCUP, this dataset provides accurate estimates of IE admissions, readmissions rate, predictors of readmissions, and associated costs.

\section{Limitations}

However, our study is limited due to its retrospective nature and its reliance on administrative data. We are unable to validate the accuracy of ICD-9-CM code documentation, which was used to determine the clinical and procedural information pertaining to the disease of interest, however, these errors are expected to be very low [21]. NRD lacks data with regards to ethnicity, socioeconomic status, and clinical data, such as echocardiographic findings of an abscess, perivalvular leaks, presence and size of vegetations, and valvular damage, to mention a few. There is also a lack of data with regards to blood cultures, antibiotic therapy duration after discharge, or compliance with medications. The microorganisms identified using ICD-9-CM codes are assumed to be the causative organisms, however, we are unable to validate these data.

\section{Conclusions}

In the United States, there is a slight decrease in the incidence of IE hospitalizations between January 1, 2010, and November 30, 2014. One in four IE patients is readmitted to the hospital within 30 days. IE patients leaving AMA or having a history of drug abuse and CIED in-situ or prosthetic valve in-situ were at the highest risk for 30-day readmission. The annual cost of readmissions after IE is $\$ 58$ million. Further research is needed to investigate the impact of clinical and social measures that could decrease patients' readmissions after IE and curb the high associated costs.

\section{Additional Information}

\section{Disclosures}

Human subjects: Consent was obtained by all participants in this study. Animal subjects: All authors have confirmed that this study did not involve animal subjects or tissue. Conflicts of interest: In compliance with the ICMJE uniform disclosure form, all authors declare the following: Payment/services info: All authors have declared that no financial support was received from any organization for the submitted work. Financial relationships: All authors have declared that they have no financial relationships at present or within the previous three years with any organizations that might have an interest in the submitted work. Intellectual property info: Dr. Saba has received research support from Boston Scientific and Medtronic. All other authors report no financial or intellectual conflicts of interest related to this manuscript. Other relationships: All authors have declared that there are no other relationships or activities that could appear to have influenced the submitted work.

\section{Acknowledgements}

The results of the present study were presented at the 2019 American College of Cardiology conference in New Orleans, Louisiana, USA.

\section{References}

1. Park LP, Chu VH, Peterson G, et al.: Validated risk score for predicting 6-month mortality in infective endocarditis. J Am Heart Assoc. 2016, 5:003016. 10.1161/JAHA.115.003016

2. Rasmussen TB, Zwisler AD, Thygesen LC, Bundgaard H, Moons P, Berg SK: High readmission rates and mental distress after infective endocarditis - results from the national populationbased CopenHeart IE survey. Int J Cardiol. 2017, 235:133-140. 10.1016/j.ijcard.2017.02.077

3. Wang A, Gaca JG, Chu VH: Management considerations in infective endocarditis: a review . 
JAMA. 2018, 320:72-83. 10.1001/jama.2018.7596

4. NRD Database Documentation. Healthcare Cost and Utilization Project (HCUP) . (2017). Accessed: November 2017: https://www.hcupus.ahrq.gov/db/nation/nrd/nrddbdocumentation.jsp.

5. Toyoda N, Chikwe J, Itagaki S, Gelijns AC, Adams DH, Egorova NN: Trends in infective endocarditis in California and New York State, 1998-2013. JAMA. 2017, 317:1652-1660. 10.1001/jama.2017.4287

6. Elixhauser A, Steiner C, Harris DR, Coffey RM: Comorbidity measures for use with administrative data. Med Care. 1998, 36:8-27.

7. Moore BJ, White S, Washington R, Coenen N, Elixhauser A: Identifying increased risk of readmission and in-hospital mortality using hospital administrative data: The AHRQ Elixhauser Comorbidity Index. Med Care. 2017, 55:698-705. 10.1097/mlr.0000000000000735

8. Elixhauser Comorbidity Software, Version 3.7 . https://www.hcupus.ahrq.gov/toolssoftware/comorbidity/comorbidity.jsp.

9. Pasupula DK, Rajaratnam A, Rattan R, et al.: Trends in hospital admissions for and readmissions after cardiac implantable electronic device procedures in the United States: an analysis from 2010 to 2014 using the National Readmission Database. Mayo Clin Proc. 2019, 94:588-598. 10.1016/j.mayocp.2018.10.028

10. United States Department of Labour, Bureau of Labor Statistics . https://data.bls.gov/pdq/SurveyOutputServlet.

11. Baddour LM, Wilson WR, Bayer AS, et al.: Infective endocarditis: diagnosis, antimicrobial therapy, and management of complications: a statement for healthcare professionals from the Committee on Rheumatic Fever, Endocarditis, and Kawasaki Disease, Council on Cardiovascular Disease in the Young, and the Councils on Clinical Cardiology, Stroke, and Cardiovascular Surgery and Anesthesia, American Heart Association: endorsed by the Infectious Diseases Society of America. Circulation. 2005, 111:394-434. 10.1161/CIRCULATIONAHA.105.165564

12. Baddour LM, Wilson WR, Bayer AS, et al.: Infective endocarditis in adults: diagnosis, antimicrobial therapy, and management of complications: a scientific statement for healthcare professionals from the American Heart Association. Circulation. 2015, 132:14351486. 10.1161/CIR.0000000000000296

13. Nishimura RA, Carabello BA, Faxon DP, et al.: ACC/AHA 2008 guideline update on valvular heart disease: focused update on infective endocarditis: a report of the American College of Cardiology/American Heart Association Task Force on practice guidelines: endorsed by the Society of Cardiovascular Anesthesiologists, Society for Cardiovascular Angiography and Interventions, and Society of Thoracic Surgeons. Circulation. 2008, 118:887-896. 10.1161/CIRCULATIONAHA.108.190377

14. Pant S, Patel NJ, Deshmukh A, et al.: Trends in infective endocarditis incidence, microbiology, and valve replacement in the United States from 2000 to 2011. J Am Coll Cardiol. 2015, 65:2070-2076. 10.1016/j.jacc.2015.03.518

15. Ahmad S, Munir MB, Sharbaugh MS, Althouse AD, Pasupula DK, Saba S: Causes and predictors of 30-day readmission after cardiovascular implantable electronic devices implantation: Insights from Nationwide Readmissions Database. J Cardiovasc Electrophysiol. 2018, 29:456-462. 10.1111/jce.13396

16. Fingar K, Washington R: Trends in hospital readmissions for four high-volume conditions, 2009-2013. Statistical Brief \#196 [EPub]. Healthcare Cost and Utilization Project (HCUP) Statistical Briefs. 2015,

17. Fingar K, Barrett ML, Joanna J: A comparison of all-cause 7-day and 30-day readmissions, 2014. Statistical brief \#230 [EPub]. Healthcare Cost and Utilization Project (HCUP) Statistical Briefs. 2017,

18. National Institute on Drug Abuse. Nationwide trends . 2015, Accessed: January 31, 2019: https://www.drugabuse.gov/publications/drugfacts/nationwide-trends.

19. Iversen K, Ihlemann N, Gill SU, et al.: Partial oral versus intravenous antibiotic treatment of endocarditis. N Engl J Med. 2019, 380:415-424. 10.1056/NEJMoa1808312

20. Centers for Medicare \& Medicaid Services. Hospital Readmissions Reduction Program (HRRP). https://www.cms.gov/medicare/medicare-fee-for-servicepayment/acuteinpatientpps/readmissions-reduction-program.html.

21. Lorence DP, Ibrahim IA: Benchmarking variation in coding accuracy across the United States . 


\section{Cureus}

J Health Care Finance. 2003, 29:29-42. 